\title{
Temporal Analysis of the Millisecond X-ray Pulsar SAX J1808.4-3658 During the 2000 Outburst
}

\author{
Alessandro Papitto*, Luciano Burderi ${ }^{\dagger}$, Tiziana Di Salvo**, Maria Teresa \\ Menna* and Luigi Stella* \\ * Osservatorio Astronomico di Roma, via Frascati 33, 00040 Monteporzio Catone (Rome, Italy) \\ ${ }^{\dagger}$ Univ.degli Studi di Cagliari, Dip.di Fisica, SP Monserrato-Sestu km 0.7, 09042 Monserrato, Italy \\ ${ }^{* *}$ Dipartimento di Scienze Fisiche e Astronomiche, Universitá di Palermo, via Archirafi 36, 90123, \\ Palermo, Italy
}

\begin{abstract}
We report a temporal analysis of the millisecond X-ray Pulsar SAX J1808.4-3658 during the 2000 outburst, observed with RXTE.

The observed maximum luminosity was approximately a factor of ten lower than in the other outbursts exhibited by the source, and this low flux level forced us to use a technique based on the $\chi^{2}$ obtained with an epoch folding search to discriminate between different possible orbital solutions, in order to correct the data for the orbital motion.

In the subsequent searches for periodicities we clearly detected the $401 \mathrm{~Hz}$ pulsation in at least two observations, but in the faintest the pulsed fraction varied from $20 \%$ ca. to the absence of signs of coherent pulsation at all, while the measured flux remained at an almost constat level. This erratic behaviour is discussed in the context of the centrifugal inhibition of accretion.
\end{abstract}

Keywords: pulsars:general - pulsars: individual(SAX J1808.4-3658 ) - stars:magnetic fields stars: neutron - X-rays: binaries

PACS: <Missing classification>

\section{INTRODUCTION}

The X-ray transient SAX J1808.4-3658 was discovered in September 1996 when it exhibited an outburst detected by the BeppoSAX Wide Field Cameras (in't Zand et al. 1998). The source had a maximum luminosity of $\sim 2.5 \times 10^{36} \mathrm{erg} \mathrm{s}^{-1}(3-25 \mathrm{keV})$ and also showed three type-I X-ray bursts that led to the identification of the compact object as a neutron star (NS) and to the derivation of a distance of about $2.5 \mathrm{kpc}$ (in 't Zand et al. 1998;2001).

The source was found in outburst again in 1998 April, when the high temporal resolution of the Proportional Counter Array (PCA) on board the Rossi X-ray Timing Explorer (RXTE) made it possible for Wijanands \& van der Klis (1998) to discover coherent 401 $\mathrm{Hz}$ pulsations, making this source the first known accretion-driven millisecond pulsar. The peak luminosity was about $3.5 \times 10^{36} \mathrm{erg} \mathrm{s}^{-1}$ in the $3-150 \mathrm{keV}$ band. Timing analysis performed on data collected over the period 1998 April 11-18 made it possible for Chakrabarty \& Morgan (1998, hereafter CM98) to determine the orbital parameters of the system, such as the $\sim 2 \mathrm{hr}$ orbital period.

SAX J1808.4-3658 was detected in outburst for the third time on the 2000 January $21^{\text {st }}$ (van der Klis et al. 2000), and observed again with RXTE/PCA, but this time at 
a flux level of about a tenth of the previously observed fluxes. Nevertheless it is worth noting that in the days preceeding the detection the source could not be observed due to solar constrainsts. The maximum luminosity reached by the source was $\sim 2.5 \times$ $10^{35} \mathrm{erg} \mathrm{s}^{-1}(3-25 \mathrm{keV})$ on the $2^{\text {nd }}$ of February, but its proximity to the Sun at that time made it impossible to determine the exact moment of the start of the outburst, so that the peak luminosity could have been significantly higher. The behaviour of the Xray luminosity, towards the end of the 2000 outburst, was highly erratic with dramatic variations by a factor $\sim 20$ on time scales of 5 hours and by an even larger factor (up to 1000) on time scales of a few days (Wijnands et al. 2001).

This kind of behaviour was again exhibited by the same source in the later stages of the 2002 October outburst (Wijnands 2004). In the first few weeks of this outburst the light curve resembled very nearly the 1998 one, with a similar peak luminosity and a steady decay that after about two weeks became steeper; five days later the flux raised again, reaching about one tenth of the peak level. Then the source entered in a low level activity state, with luminosity swings similar to those of the 2000 outburst, suggesting on one hand that a true outburst may have occurred, although unobserved, in 2000, and on the other that this low level activity state could be typical for this source.

\section{OBSERVATIONS}

Throughout this work we used the Target of Opportunity pubblic domain data of the PCA on board RXTE (Bradt et al. 1993). In particular, we analyzed data from the PCA (Jahoda et al. 1996), which is composed of a set of five xenon proportional counters operating in the $2-60 \mathrm{keV}$ energy range with a total effective area of $\sim 7000 \mathrm{~cm}^{2}$. We considered all the available PCA observations of SAX J1808.4-3658 taken during the 2000 outburst covering the period January 21st - March 1st, focusing then our attention on the two brighter observations of this outburst (February 2nd and 8th), in which the spin modulation was strong enough to be analyzed. As we had to perform a temporal analysis on these data, first of all we reported all the events arrival times to the solar system barycenter, using the JPL's ephemerides DE200. In order to extract the light curves we considered data taken in Standard 2 Mode (129 channels; $16 \mathrm{~s}$ in resolution), while event mode data $E \_125 U S \_64 M \_0 \_1 S$ (64 channels; $122 \mu s$ resolution) were used to perform temporal analysis. The data were background subtracted and analyzed according to the RXTE Cook Book recipes, using FTOOLS ver.5.2.

\section{ORBITAL MOTION}

The orbital motion of the source relative to the observer, determines a Doppler shift of the spin ferquency of the NS. In other terms the difference between the emission and the arrival times of the X-ray photons depends on the position of the source with respect to the line of nodes of the binary system. Neglecting any second order term in expanding $\left(t_{e m}-t_{\text {arr }}\right)$ the relation between emission and arrival times is:

$$
t_{\text {em }}=t_{\text {arr }}-\frac{a \operatorname{sini}}{c} \sin \left[\frac{2 \pi}{P_{\text {orb }}}\left(t_{\text {arr }}-T^{*}\right)\right]
$$


where the $t_{a r r}$ 's are the photon arrival times at the line of barycenter of the Solar System, the $t_{e m}$ 's are the emission times referred to barycenter of the binary system, $\frac{a s i n i}{c}$ is the projected semimajor axis of the orbit, and $T^{*}$ is the time of ascending node passage. Therefore with an observational estimate of the orbital parameters $a \sin i, P_{\text {orb }}$ and $T^{*}$ we can correct the arrival times of the photons emitted by the compact object for the delays induced by the orbital motion.

The high temporal resolution RXTE data of SAX J1808.4-3658 span a period of $\Delta T_{\text {data }} \sim 1.8 \mathrm{yr}$, from April 1998 to February 2000, during which SAX J1808.4-3658 performed $N_{\max } \sim 8000$ orbital cycles and $n_{\max } \sim 22$ billion spin cycles. In order to perform a timing analysis over the whole $\Delta T_{\text {data }}$ we must unambigously associate $n$, i.e. the number of elapsed spin cycles since the 1998 outburtst, to any given $t_{\text {arr }}$. Unfortunately, even neglecting any error on the spin period estimate $P_{\text {spin }}$, induced by the errors in the orbital correction, this association is not possible as the uncertainity on the CM98 estimate of the spin period $\left(\sigma_{\text {Pspin }} \sim 5 \times 10^{-12}\right)$ is at least one order of magnitude larger than the one needed to allow it $\left(\sigma_{\text {Pspin }} \leq P_{\text {spin }} / n_{\max } \sim 1 \times 10^{-13} \mathrm{~s}\right.$. $)$, thus ruling out the possibility of making an overall timing analysis over $\Delta T_{\text {data }}$.

However, the large value of $N_{\max }$, that becomes even larger considering the 2002 outburst data too, suggested us to develop a method of timing of the orbital period $P_{\text {orb }}$ in order to improve the orbital period estimate of CM98. Our idea is to estimate the time of passage at a given point in the orbit, e.g. the ascending node $T^{*}$, for any given orbital cycle $N$, and to fit these times $T^{*} \mathrm{~s} v s$ the corresponding $N$ to improve the estimate of $P_{\text {orb }}$ and to give a value (or an upper limit) for the orbital period derivative $\dot{P}_{\text {orb }}$.

Our strategy to estimate the values of $T^{*}$ relies on the fact that in correcting the photon arrival times with equation 1 , using a certain set of orbital parameters, the more they are far from the 'real' ones, the more the resulting pulse profiles obtained with an Epoch Folding will be smeared, hence with a smaller pulse fraction.

The experimental estimate of each $T^{*}$ can be thus achieved by correcting the light curves of interest with different values of $T^{*}$, choosing as our estimate of this parameter the one that gives the maximum $\chi^{2}$ in an epoch folding search.

This technique is discussed in details in Papitto et al. 2005 and its application to the three outburst of SAX J1808.4-3658, allowed us to determine an unique orbital solution valid over the entire $5 y r$ period (1998 april - 2002 october) for which high temporal resolution data were available. We revised the previous estimate of the orbital period, $P_{\text {orb }}=7249.1569(1) \mathrm{s}$, and reduced the corresponding error by 1 order of magnitude with respect to that previuosly reported in CM98. Moreover we could get to the first constraint on the orbital period derivative, $-6.6 \times 10^{-12}<\dot{P}_{\text {orb }}<+0.8 \times 10^{-12} s^{-1}$. These orbital parameters allowed us to produce, via a folding technique, pulse profiles at any given time.

\section{PULSE PROFILES DURING 2000 FEBRUARY 8 OBSERVATION}

We focused our attention on the two brighter observations of the 2000 outburst in which the count rate was high enough to allow an analysis of the pulse profiles.

We initially performed an epoch folding search on these data dividing each cycle in 
5 phase bins and a Gaussian fit of the resulting $\chi^{2}$ vs. spin period curve resulted gave an estimate of $P_{S P I N}=0.002493919711(2) s$. The average pulse profiles, obtained by folding the light curves of interest around this value of the spin period and dividing each spin cycle into 20 phase bins, are shown in Figure 1; the average pulse fractions, evaluated as $p f=\left(I_{M A X}-I_{M I N}\right) / I_{M A X}$, with $I_{M A X}$ and $I_{M I N}$ maximum and minimum count rate respectively, were of $(11.5 \% \pm 0.5 \%)$ (February 2$)$ and $(10.4 \% \pm 0.7 \%)$ (February 8) (left panel of Fig.2).

While in the February 2 observation the pulsating actvity remained stable and clear troughout its whole duration, in the February 8 one it showed an intermittent behaviour, without any appreciable simultaneous variation in the X-ray flux. Considering $200 s$ time intervals and evaluating the $p f$ on the folded pulse profiles as before, we detected pulse fraction variations from $\sim 20 \%$ to the absence of significant coherent pulsation at all, regardless of a nearly constant luminosity. The overall behaviour of the pulse fraction and luminosity is showed in the right panel of Fig.2.

In order to search for possible correlations between the pulse fraction and the X-ray flux, we performed a Pearson's linear correlation test on the roughly linearly increasing part of this template. This test measures the strenght of a supposed linear relationship between two variables in terms of a coefficient $r=\operatorname{cov}(X, Y) /\left(\sigma_{X} \sigma_{Y}\right),|r| \leq 1$. We found $r=0.80$ for the points representing pulse fraction and time and $r=-0.18$ for luminosity and time, which mean, for $N=12$ points, that the probability the pulsed fraction is not linearly correlated with time is less than $0.2 \%$, while the the probability that luminosity is not linearly correlated with time is more than $50 \%$.

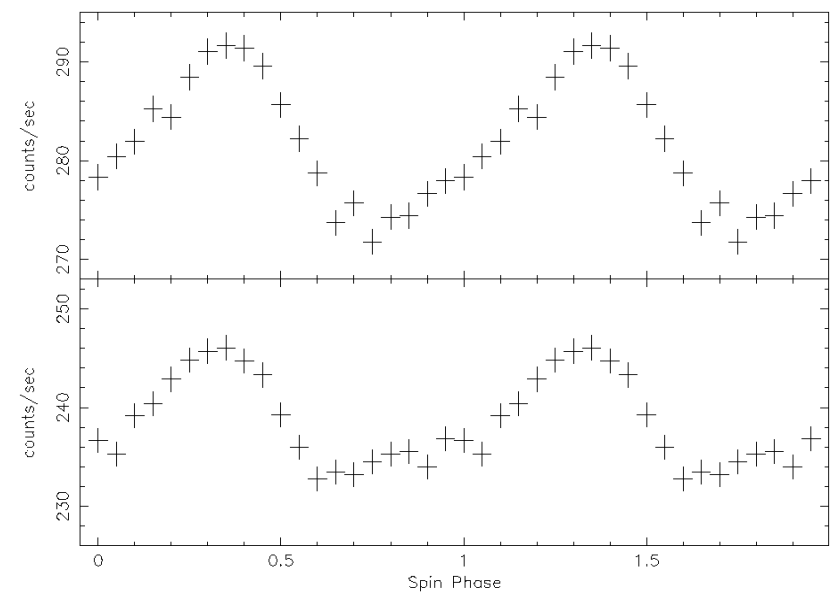

FIGURE 1. Average pulse profiles of the entire February 2 (top) and February 8 (bottom) observations. The light curves were folded around $P_{\text {SPIN }}=2.493919711 \mathrm{~ms}$ while every cycle was divided in 20 phase bins. 
Finally we searched for any spectral variations that may be associated with the disappearance of pulsations, but the source remained quite hard troughout the observations, with a behaviour reminiscent of that of an atoll sources.
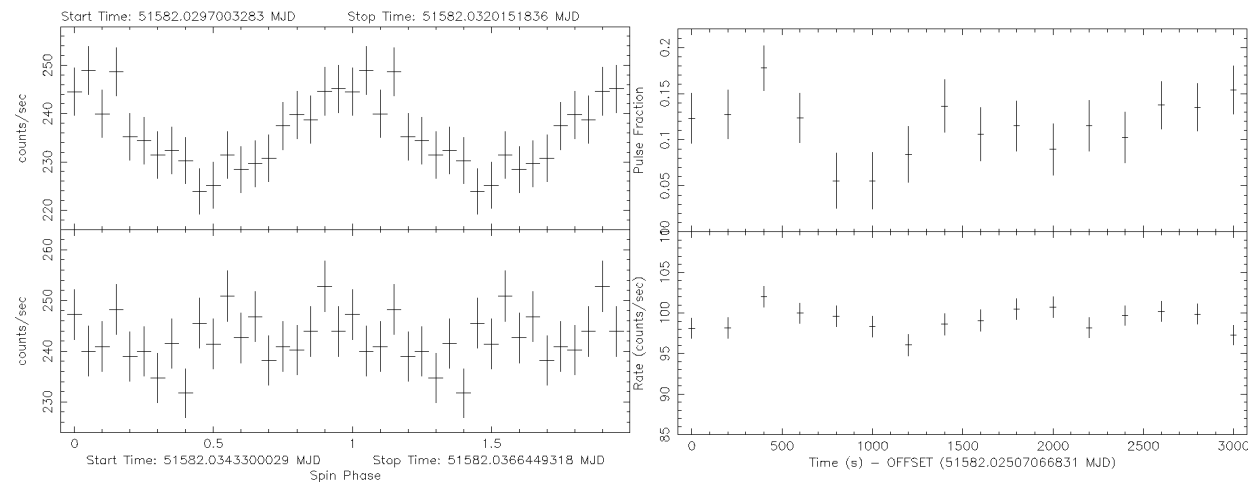

FIGURE 2. (Left):Pulse profiles in two different $200 s$ intervals of the February 8 observation. The folded period is $P_{\text {spin }}=2.493919711 \mathrm{~ms}$, obtained from an Epoch Folding Search, as discussed in text. The top panel is referred to the third interval, while the bottom one is referred to the fifth. (Right): Pulsed fraction vs. time (top panel) and corresponding count rate $(2-60 \mathrm{keV})$ (bottom panel) for the February 8 observation.

\section{DISCUSSION}

In the standard picture of disc accretion onto a neutron star of radius $R_{N S}$, whose magnetic field is strong enough to disrupt the disc at some magnetospheric radius $R_{M}>R_{N S}$, the total X-ray energy output can be divided in two terms:

$$
L_{X}=\frac{G M \dot{M}}{2 R_{M}}+G M \dot{M}\left[\frac{1}{R_{N S}}-\frac{1}{2 R_{M}}\right]
$$

The first represents the emission from matter in the disc that gradually spirals in, down to the magnetospheric radius, while the second arises from matter infall onto the NS polar caps. A fraction $f$ of the second term, depending mainly on the geometry of accretion path and on scattering processes near the surface, will be actually pulsed. It is then possible to express the pulsed fraction as:

$$
p f=\frac{f \times G M \dot{M}\left[\frac{1}{R_{N S}}-\frac{1}{2 R_{M}}\right]}{\frac{G M \dot{M}}{2 R_{M}}+G M \dot{M}\left[\frac{1}{R_{N S}}-\frac{1}{2 R_{M}}\right]}=f \times\left(1-\frac{R_{N S}}{2 R_{M}}\right)
$$

Expressing the magnetospheric radius as a roughly constant fraction of the Alfven radius $\left(R_{A} \sim \dot{M}^{-2 / 7}\right)$, we then expect the pulse fraction to be a slowly decreasing function of $\dot{M}$ and an increasing function of $f$. 
During 2000, February 8 observations SAX J1808.4-3658 showed strong variations in pulsed fraction, and in particular a sharp cut-off of the pulsating activity, while the $\mathrm{X}$-ray flux, and hence the mass accretion rate, remained at an almost constant level. According to the simple picture we outlined before, these variations can be accounted only by changes in the parameter $f$ or by a marginal decrease in the mass accretion rate that triggers a different accretion state.

The first hypotesis involves rather polar caps size than scattering environment variations, as no significant spectral evolution was detected during the disappearance of pulses. It has to be noted that fluctuations in the polar caps extent and structure were also invoked by C.Markwardt (IB2004), in explaining the apparent alternating spin up/down beahviour, exhibited by the same source during the October 2002 outburst.

The intermittent behaviour of pulsations can even be interpreted according to the propeller scenario (Illarionov \& Sunyaev 1975), in which the accretion of matter onto the NS surface is inhibited by the onsetting of a centrifugal barrier, when the mass accretion rate drops below a critical value and the magnetospheric radius equals the corotation radius. Subsequently Spruit \& Tamm (1993) have pointed out how the condition $R_{M}=$ $R_{C O}$ is energetically insufficient to unbind all the incoming matter from the gravitational influence of the NS, and then that there must be an intermediate accretion state, for $R_{M} \sim R_{C O}$, in which mass accretion is neither allowed in the standard way nor halted by the centrifugal barrier, but rather proceeds in a cyclic way. RXTE observed the source again approximately 40 hours after the Feb8 observation, but this time was below the detection threshold $\left(\mathrm{few} \times 10^{33} \mathrm{ergs}^{-1}\right)$; we can therefore interpret the disappearance of pulsations as an intermediate phase of the onsetting of centrifugal barrier, in which the pulses starts to become less clear, but the luminosity did not had the expected cut off still. However a detailed coverage of these luminosity swings is missing and we could not even study the possibility of a connection between these pulsed fraction variations and the orbital motion, as already observed by Kirsch et al. (2004) on another source of this class, XTE J1807-294.

\section{REFERENCES}

1. Bradt, H., Rotschild, R.E. \& Swank, J.H. 1993, A\&AS, 97, 355.

2. Chakrabarty, D. \& Morgan, E.H. 1998, Nature, 394, 346 (CM98).

3. Ilarionov, A.F. \& Sunyaev, R.A. $1975, A \& A, 39,185$ I

4. in't Zand, J.J.M. et al. 1998, A\&A, 331, L25.

5. in't Zand, J.J.M. et al. 2001, $A \& A, 372,916$.

6. Jahoda, K. et al. 1996, Proc.SPIE 2808, 59.

7. Kirsch, M.G.F. et al. 2004, $A \& A, 423 \mathrm{~L}, 9$

8. Papitto, A., Menna, M.T., Burderi, L., Di Salvo, T., D’Antona, F. \& Robba, N.R. 2005, ApJL, 621.

9. Spruit, H.C. \& Tamm, R.E. 1993, ApJ, 402, 593S

10. van der Klis, M. et al. 2000, IAU Circ., 7358, 3.

11. Wijnands, R. \& van der Klis, M. 1998, Nature, 394, 344.

12. Wijnands, R., et al. 2001, ApJ, 560, 892.

13. Wijnands, R. 2004, in AIP Conf.Proc. 714, X-Ray Timing 2003: Rossi and beyond, ed. P.Kaaret, F.K.Lamb \& J.H. Swank (Melville: AIP), 209 
Copyright of AIP Conference Proceedings is the property of American Institute of Physics. The copyright in an individual article may be maintained by the author in certain cases. Content may not be copied or emailed to multiple sites or posted to a listserv without the copyright holder's express written permission. However, users may print, download, or email articles for individual use. 